\title{
BMJ Open Characteristics of urinary tract infection pathogens and their in vitro susceptibility to antimicrobial agents in China: data from a multicenter study
}

\author{
Lu-Dong Qiao, ${ }^{1}$ Shan Chen, ${ }^{1}$ Yong Yang, ${ }^{2}$ Kai Zhang, ${ }^{3}$ Bo Zheng, ${ }^{4}$ \\ Hong-Feng Guo, ${ }^{5}$ Bo Yang, ${ }^{6}$ Yuan-Jie Niu, ${ }^{7}$ Yi Wang, ${ }^{8}$ Ben-Kang Shi, ${ }^{9}$ \\ Wei-Min Yang, ${ }^{10}$ Xiao-Kun Zhao, ${ }^{11}$ Xiao-Feng Gao, ${ }^{12}$ Ming Chen, ${ }^{13}$ Ye $\operatorname{Tian}^{14}$
}

To cite: Qiao L-D, Chen S, Yang $\mathrm{Y}$, et al. Characteristics of urinary tract infection pathogens and their in vitro susceptibility to antimicrobial agents in China: data from a multicenter study. BMJ Open 2013;3:e004152. doi:10.1136/ bmjopen-2013-004152

- Prepublication history for this paper is available online. To view these files please visit the journal online (http://dx.doi.org/10.1136/ bmjopen-2013-004152).

Received 30 September 2013 Revised 18 November 2013 Accepted 19 November 2013

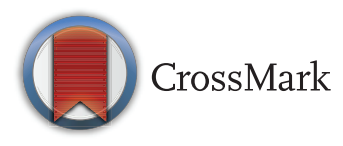

For numbered affiliations see end of article.

Correspondence to Professor Ye Tian; tianye166@126.com

\section{ABSTRACT}

Objective: This study assessed the characteristics of pathogens identified in clinical isolates from patients with urinary tract infection (UTI) and their in vitro sensitivity to commonly used antibiotics in the clinical setting in

China.

Design and setting: Multicenter study was conducted between January and December 2011 in 12 hospitals in China.

Participants: Urine samples were collected from 356 symptomatic patients treated in the study hospitals for acute uncomplicated cystitis, recurrent UTI or complicated UTI.

\section{Primary and secondary outcome measures:}

Minimal inhibitory concentrations (MICs) were measured using broth microdilution according to the Clinical and Laboratory Standards Institute 2011 guidelines. Thirteen antimicrobial agents were tested: fosfomycin tromethamine, levofloxacin, moxifloxacin, cefdinir, cefixime, cefaclor, cefprozil, cefuroxime, amoxicillin/clavulanic acid, cefotaxime, azithromycin, nitrofurantoin and oxacillin. Escherichia coli isolates were screened and extended spectrum $\beta$-lactamases (ESBL) production was confirmed by a double-disk synergy test.

Results: 198 urine samples were culture-positive and 175 isolates were included in the final analysis. E coli was detected in $50 \%$ of cultures, followed by Staphylococcus epidermidis (9\%), Enterococcus faecalis $(9 \%)$ and Klebsiella pneumoniae $(5 \%)$. The detection rate of ESBLproducing $E$ coli was $53 \%$. Resistance to levofloxacin was the most common among all the isolates. Nitrofurantoin and fosfomycin tromethamine had the greatest activity against $E$ coli; overall, $92 \%$ and $91 \%$ of isolates were susceptible to these antimicrobials. $E$ faecalis had the highest susceptibility rates to fosfomycin tromethamine (100\%).

Conclusions: The most frequently identified pathogens in our patients were ESBL-producing $E$ coli and $E$ faecalis. Fosfomycin tromethamine and nitrofurantoin showed a good antimicrobial activity against UTI pathogens. They may represent good options for the empiric treatment of patients with UTI.

\section{Strengths and limitations of this study}

- This study was conducted in 12 major clinical research centres from China. Antibiotic susceptibility was tested with 13 antimicrobial agents that are frequently used in the clinical practice in China.

- A strength of the study is correct subspecification of coagulase negative staphylococci, enterococci and streptococci.

- For several pathogens, due to the relatively low number of tested isolates, differences in susceptibility patterns between various urinary tract infection (UTI) types could not be assessed. No distinction was made between communityacquired and healthcare-associated UTIs.

\section{INTRODUCTION}

Urinary tract infections (UTIs) are common infectious diseases in clinical practice. An estimated 150 million people worldwide are diagnosed with a UTI each year, ${ }^{1}$ and $40-50 \%$ of women present a UTI at least once in their lifetime. $^{2-5}$ The results of a survey performed in the USA estimated that a UTI episode was associated with an average of 6.1 days with symptoms, 2.4 days of reduced activity and 0.4 days of bed rest, thus generating an estimated annual cost (direct and indirect) of 1.6 billion dollars. $^{6-8}$ In China, UTIs account for 9.39$50 \%$ of nosocomial infections. ${ }^{910}$ Most cases of UTI are caused by Gram-negative bacilli, with Escherichia coli accounting for over $90 \%$ of uncomplicated UTIs. ${ }^{11}$ Uncomplicated infections can be treated with short courses of antibiotics, while complicated UTIs require longer and more intensive courses of antibiotics. However, resistance to the commonly used antibiotics is increasing and making treatment more difficult. 
In China, clinical isolates of $E$ coli have been shown to have resistance rates as high as $20.6-27.9 \%$ to amoxicillin/ clavulanic acid, $64.7-74 \%$ to ciprofloxacin and $71.1-80.1 \%$ to piperacillin. ${ }^{12} 13$ Many cases in which UTIs are resistant to conventional treatment have been associated with $E$ coli isolates producing extended spectrum $\beta$-lactamases (ESBLs). The emergence of these ESBL-producing isolates makes clinical treatment even more difficult. This study assesses the distribution of pathogens of acute uncomplicated cystitis, recurrent UTI or complicated UTI and their in-vitro sensitivity to commonly used antibiotics in the clinical treatment of these infections. These findings will be informative for physicians in their decision-making in empirical medicine, thus contributing to the prevention and mitigation of the increase in drug resistance.

\section{PATIENTS AND METHODS}

\section{Source of isolates}

Enrolled patients were between 18 years old and 70 years old, suffered from acute uncomplicated cystitis, recurrent UTI or complicated UTI, were symptomatic and were treated in the urology department in 1 of 12 clinical research centres between 26 January and 7 December 2011. The study was conducted in accordance with the Declaration of Helsinki and approved by the ethics committee of Beijing Tongren Affiliated Hospital of Capital Medical University. The study patients signed an informed consent form before any study procedure was conducted. Midstream urine specimens for bacterial culture were collected before treatment. Colony counts higher than or equal to $10^{4}$ colony forming units $(\mathrm{CFU}) / \mathrm{mL}$ were considered significant.

\section{Antibiotics and reagents}

The following antimicrobial agents were used for susceptibility testing: oxacillin, amoxicillin, cefaclor, cefuroxime and nitrofurantoin, obtained from the National Institute for Food and Drug control (Beijing, China); levofloxacin from Daiichi Sankyo (Beijing, China); moxifloxacin and fosfomycin tromethamine produced by Shanxi Qianyuan Pharmaceutical Co., Ltd (Shanxi, China); cefdinir from Jinkang, Tianjin Pharmaceutical Group (Tianjin, China); cefixime from Guangzhou Baiyunshan Pharmaceutical Factory (Guangzhou, China); cefprozil from Bristol-Myers Squibb (Shanghai, China); cefotaxime from Sanjiu Group Shenzhen Jiuxin Pharmaceutical Co., Ltd (Shenzhen, China); azithromycin from Pfizer (Dalian, China) and clavulanic acid from GlaxoSmithKline (Tianjin, China).

The amoxicillin/clavulanic acid combination was used at a ratio of 2:1. For the cefotaxime/clavulanic acid combination, clavulanic acid concentration was kept constant at $4 \mathrm{mg} / \mathrm{L}$. Susceptibility medium (Mueller-Hinton $(\mathrm{MH})$ ), ceftazidime (CAZ, $30 \mu \mathrm{g}$ ) (used to detect ESBLs), amoxicillin/clavulanic acid (AMC, $20 \mu \mathrm{g} / 10 \mu \mathrm{g}$ ) and cefotaxime (CTX, $30 \mu \mathrm{g})$ susceptibility paper disks were purchased from Thermo Fisher Biochemicals (Beijing) Ltd (Beijing, China).

\section{Tested isolates}

The sensitivity test was standardised using the following reference isolates: Staphylococcus aureus ATCC 29213, $E$ faecalis ATCG 29212, E coli ATCC 25922 and Pseudomonas aeruginosa ATCC 27853, originating from the American-type culture collection.

Clinical isolates were identified in the urine samples collected in the clinical research centres participating in the study. Medium and incubation conditions: $S$ aureus, Enterococcus spp and Gram-negative bacteria were incubated in $\mathrm{MH}$ medium at $35^{\circ} \mathrm{C}$ for $16-20 \mathrm{~h}$. Susceptibility of staphylococci to oxacillin was assessed using $\mathrm{MH}$ agar supplemented with $2 \% \mathrm{NaCl}$ solution, and $24 \mathrm{~h}$ additional incubation. Streptococci were incubated in blood culture medium ( $5 \%$ defibrinated sheep blood added to $\mathrm{MH}$ medium) at $35^{\circ} \mathrm{C}$ in $5 \% \mathrm{CO}_{2}\left(\mathrm{CO}_{2}\right.$ incubator $)$ for 20-24 h.

\section{Minimum inhibitory concentration measurement}

For minimal inhibitory concentration (MIC) measurement, a standard plate count and double dilution method was used. Bacterial suspensions to be tested were prepared by inoculation with $10^{4} \mathrm{CFU}$ of each bacterium, using a multipoint inoculator. The MIC of the antimicrobial agents was measured for a variety of pathogens.

\section{Processing of the results}

MIC50 and MIC90 values were calculated, as well as the bacterial resistance rate, the intermediate rate and the sensitivity rate according to the critical concentration standards for antimicrobial drugs promulgated by the Clinical and Laboratory Standards Institute in 2011. ${ }^{14}$ ESBL-producing E coli, Klebsiella pneumoniae and Proteus mirabilis were identified using a double-disc synergy test. ${ }^{15}$

\section{RESULTS}

\section{Distribution of isolates}

A total of 356 patients were enrolled and provided a urine sample. Of these, $198(55.6 \%)$ tested positive for significant bacteriuria. In 23 urine samples, the clinical pathogens could not be identified without performing additional tests and were excluded from the analysis. A total of 175 isolates with identified pathogens were included in the final analysis: $116(66.3 \%)$ Gram-negative bacteria and 59 (33.7\%) Gram-positive bacteria. E coli was the most frequently identified Gram-negative bacteria (in 87 isolates $(49.7 \%))$. The most frequently identified Gram-positive pathogens were: Staphylococcus spp (in 30 isolates (17.1\%)), Enterococcus spp (in 19 isolates (10.9\%)) and Streptococcus spp (in 10 isolates (5.7\%)); table 1).

Of the 175 isolates, $124(70.9 \%)$ were from women, with $E$ coli and $K$ pneumoniae being the most frequently identified. In men, the most frequently identified pathogens were $E$ coli and $E$ faecalis (table 2).

Ninety-five isolates were from patients with acute uncomplicated cystitis, 42 from patients with recurrent UTIs and 37 from patients with complicated UTIs (table 3 ). 
Table 1 Number and proportion of isolated pathogens from midstream urine species

\begin{tabular}{|c|c|c|c|c|c|}
\hline \multicolumn{3}{|l|}{$\begin{array}{l}\text { Gram-negative bacteria } \\
n=116\end{array}$} & \multicolumn{3}{|l|}{$\begin{array}{l}\text { Gram-positive bacteria } \\
\mathrm{n}=59\end{array}$} \\
\hline Bacterium & $\mathbf{N}$ & Per cent ${ }^{\star}$ & Bacterium & $\mathbf{N}$ & Per cent ${ }^{\star}$ \\
\hline Escherichia coli & 87 & 49.71 & Staphylococcus aureus & 4 & 2.29 \\
\hline Klebsiella pneumoniae & 9 & 5.14 & Staphylococcus epidermidis & 16 & 9.14 \\
\hline Proteus mirabilis & 6 & 3.43 & Staphylococcus hominis & 5 & 2.86 \\
\hline Proteus vulgaris & 2 & 1.14 & Staphylococcus saprophyticus & 2 & 1.14 \\
\hline Proteus penneri & 1 & 0.57 & Staphylococcus haemolyticus & 1 & 0.57 \\
\hline Enterobacter cloacae & 2 & 1.14 & Staphylococcus schleiferi & 1 & 0.57 \\
\hline Citrobacter freundii & 2 & 1.14 & Staphylococcus warneri & 1 & 0.57 \\
\hline Citrobacter koseri & 1 & 0.57 & Enterococcus faecalis & 15 & 8.57 \\
\hline Serratia marcescens & 1 & 0.57 & Enterococcus faecium & 3 & 1.71 \\
\hline Pseudomonas aeruginosa & 4 & 2.29 & Enterococcus gallinarum & 1 & 0.57 \\
\hline \multirow[t]{5}{*}{ Pseudomonas putida } & 1 & 0.57 & Streptococcus agalactiae & 5 & 2.86 \\
\hline & & & Streptococcus oralis & 2 & 1.14 \\
\hline & & & Streptococcus acidominimus & 1 & 0.57 \\
\hline & & & Streptococcus haemolyticus & 1 & 0.57 \\
\hline & & & Streptococcus mitis & 1 & 0.57 \\
\hline
\end{tabular}

\section{E coli antibiotic resistance}

Of the $87 \mathrm{E}$ coli isolates, $49.4 \%$ were resistant to levofloxacin, $50.6 \%$ were resistant to second-generation cephalosporin and $57.5 \%$ to third-generation cephalosporin. Forty-six E coli isolates $(52.9 \%)$ were ESBL producers (table 4).

Nitrofurantoin and fosfomycin tromethamine had the greatest activity against E coli; overall, $92 \%$ and $91 \%$ of isolates were susceptible to these antimicrobials. The percentages of isolates positive for $E$ coli by UTI type, and the proportion of ESBL-producing isolates are presented in table 3 .

\section{Susceptibility results for $K$ pneumoniae and Proteus spp}

Overall, nine isolates were identified as $K$ pneumoniae $(5.1 \%)$ and nine as Proteus bacilli $(5.1 \%)$. The corresponding susceptibility results are presented in table 5 .

Antibacterial activity of commonly used antibiotics against coagulase-negative staphylococci and $E$ faecalis

For coagulase-negative staphylococci, sensitivity rates ranged from $38.5 \%$ for oxacillin to $100 \%$ for nitrofurantoin and resistance rates from $0 \%$ for nitrofurantoin to $84.6 \%$ for azithromycin. For Staphylococcus epidermidis, sensitivity rates ranged from $18.8 \%$ for azithromycin to $100 \%$ for nitrofurantoin and fosfomycin tromethamine; and resistance rates ranged from $0 \%$ for nitrofurantoin and fosfomycin tromethamine to $81.2 \%$ for azithromycin. The resistance rate of $E$ faecalis against levofloxacin was $60 \%$, while the sensitivity rate to fosfomycin tromethamine and nitrofurantoin was $100 \%$. MIC values for these pathogens are presented in table 6 .

\section{DISCUSSION}

We demonstrated that, in Chinese patients with symptomatic UTI enrolled from research hospitals, E coli was the most frequent pathogen identified in men and women, accounting for $49.7 \%$ of the total isolates. Other pathogens identified were $S$ epidermidis $(9.1 \%), E$ faecalis (8.6\%), $K$ pneumoniae $(5.1 \%)$ and $P$ mirabilis $(3.4 \%)$. The results obtained in this study are similar to those of other studies conducted in China. ${ }^{16}$ Although E coli was the leading cause of UTI in men, its proportion was lower than in women. The prevalence of $E$ faecalis

Table 2 The distribution of pathogens in patients according to gender

\begin{tabular}{lcccc}
\hline & \multicolumn{2}{l}{ Women } & & Men \\
\cline { 2 - 4 } Bacteria & Number of patients & Per cent & & Number of patients \\
\hline Escherichia coli & 68 & 54.84 & 19 & 37.25 \\
Klebsiella pneumoniae & 9 & 7.26 & 0 & 0 \\
Staphylococcus epidermidis & 10 & 8.06 & 6 & 11.76 \\
Enterococcus faecalis & 5 & 4.03 & 10 & 19.61 \\
Others & 32 & 2.58 & 16 & 3.14 \\
Total & 124 & & 51 & \\
\hline
\end{tabular}


Table 3 Bacterial character of different types of UTIs

\begin{tabular}{|c|c|c|c|c|}
\hline & $\begin{array}{l}\text { Total } \\
\text { isolates }\end{array}$ & $\begin{array}{l}\text { Number of Gram-negative } \\
\text { bacteria }\left(\%^{\star}\right)\end{array}$ & $\begin{array}{l}\text { Number of } E \text { coli } \\
(\%+)\end{array}$ & $\begin{array}{l}\text { Number of ESBL positive } E \\
\text { coli }(\% \neq)\end{array}$ \\
\hline $\begin{array}{l}\text { Acute uncomplicated } \\
\text { cystitis }\end{array}$ & 96 & $68(70.83)$ & $51(53.13)$ & $25(49.02 \%)$ \\
\hline Recurrent UTIs & 42 & 29 (69.05) & $21(50.00)$ & $12(57.14 \%)$ \\
\hline Complicated UTIs & 37 & $19(51.35)$ & $15(40.54)$ & $9(60.00 \%)$ \\
\hline
\end{tabular}

infections was higher in men than in women. The infection rate with Gram-negative bacteria was higher in patients with uncomplicated cystitis than in patients with recurrent or complicated UTI, while the infection rate with Gram-positive bacteria showed an inverse trend.

E coli is the most common Gram-negative bacteria identified in UTIs. ${ }^{17} 18$ In our study, the resistance rate of this pathogen to fluoroquinolones (levofloxacin) and to second-generation and third-generation cephalosporin was high, ranging from $49.4 \%$ to $57.5 \%$. This is similar to the rates of antibiotic resistance previously reported in Chinese populations. ${ }^{19} 20$ The clinical trials conducted worldwide have reported resistance rates of up to $80 \%$ against fluoroquinolones ${ }^{20}$ and up to $75 \%$ against cephalosporin among uropathogenic E coli. ${ }^{19}$ An important mechanism of antibiotic resistance among pathogens is through ESBL production. ESBL hydrolyses oxyimino- $\beta$-lactams such as cephalosporin and monobactum. ${ }^{19} \mathrm{E}$ coli is one of the main ESBL-producing pathogens. ESBL production is transmitted from one bacterium to another through plasmids. These plasmids can carry multiple drug-resistant genes against aminoglycosides, quinolones and sulfamethoxazole at the same time. ${ }^{21}$ The phenomenon of multidrug resistance has brought tremendous difficulties to the clinical treatment of infection due to limited therapeutic options. ${ }^{16}{ }^{22}$ Haishen et $a t^{23}$ showed that ESBL-producing $E$ coli have a high resistance rate, ranging from $28.6 \%$ to $85.7 \%$ against the majority of antibiotics (excluding sulfamethoxazole), which is $20 \%$ to $80 \%$ higher than in E coli that do not produce ESBLs.

An increasing prevalence of ESBL-positive bacteria isolated from UTI patients has made the empirical treatment of these diseases difficult. Consequently, carbapenems have been increasingly prescribed as an empirical treatment for complicated UTIs, thus promoting the selection of drug-resistant bacteria and an increased prevalence of flora imbalance and fungal infections. In our study, the proportion of $E$ coli varied with the UTI type: the prevalence of $E$ coli overall and of EBSL-producing $E$ coli was lower in isolates from cases diagnosed with acute uncomplicated cystitis, compared with recurrent and complicated UTIs. The prevalence of EBSL-producing $E$ coli reached $60 \%$ in isolates from cases diagnosed with complicated UTI. Susceptibility results showed that the resistance rate of ESBL-producing $E$ coli isolates against commonly used antimicrobial drugs was higher than the rate observed in

Table 4 Antibacterial activity, bacterial sensitivity, intermediate sensitivity and resistance to antibiotics commonly used against ESBL-negative and ESBL-positive Escherichia coli

\begin{tabular}{|c|c|c|c|c|c|c|c|c|c|c|}
\hline \multirow[b]{2}{*}{ Antimicrobial drug } & \multicolumn{5}{|c|}{$\begin{array}{l}\text { ESBL-negative } E \text { coli } \\
\text { (41 isolates) }\end{array}$} & \multicolumn{5}{|c|}{$\begin{array}{l}\text { ESBL-positive } E \text { coli } \\
\text { (46 isolates) }\end{array}$} \\
\hline & $\mathrm{MIC}_{50}$ & $\mathrm{MIC}_{90}$ & $\mathbf{S} \%$ & $1 \%$ & $\mathbf{R} \%$ & $\mathrm{MIC}_{50}$ & $\mathrm{MIC}_{90}$ & $\mathbf{S} \%$ & $1 \%$ & $\mathbf{R} \%$ \\
\hline Fosfomycin tromethamine ${ }^{*}$ & 0.125 & 05 & 95.1 & 4.9 & 0.0 & 0.125 & 128 & 87.0 & 8.7 & 4.3 \\
\hline Levofloxacin & 1 & 16 & 61.0 & 4.9 & 34.1 & 8 & 32 & 21.7 & 15.3 & 63.0 \\
\hline Moxifloxacin & 0.5 & 32 & - & - & - & 16 & 64 & - & - & - \\
\hline Cefdinir & 0.25 & 2 & 87.8 & 2.4 & 9.8 & 256 & $>256$ & 4.3 & 2.2 & 93.5 \\
\hline Cefixime & 0.5 & 2 & 82.9 & 9.8 & 7.3 & 32 & 128 & 4.3 & 6.6 & 89.1 \\
\hline Cefaclor & 2 & 4 & 90.2 & 2.5 & 7.3 & $>256$ & $>256$ & 2.2 & 2.1 & 95.7 \\
\hline Cefprozil & 2 & 8 & 90.2 & 0.0 & 9.8 & $>256$ & $>256$ & 2.2 & 2.1 & 95.7 \\
\hline Cefuroxime & 4 & 16 & 82.9 & 7.3 & 9.8 & $>256$ & $>256$ & 0.0 & 0.0 & 100.0 \\
\hline Amoxicillin/clavulanic acid & 4 & 16 & 87.8 & 4.9 & 7.3 & 8 & 16 & 63.0 & 34.8 & 2.2 \\
\hline Cefotaxime & 0.062 & 0.5 & 95.1 & 0.0 & 4.9 & 64 & 256 & 2.2 & 0.0 & 97.8 \\
\hline Nitrofurantoin & 16 & 32 & 90.2 & 4.9 & 4.9 & 16 & 32 & 93.5 & 6.5 & 0.0 \\
\hline
\end{tabular}

*Determination of the breakpoint of fosfomycin tromethamine to Enterobacteriaceae (sensitivity rate $\leq 64 \mathrm{mg} / \mathrm{L}$; resistance rate $\geq 256 \mathrm{mg} / \mathrm{L}$ ). E coli, Escherichia coli; ESBL, extended spectrum $\beta$-lactamases; I, intermediate; MIC, minimal inhibitory concentration; R, resistant; S, susceptible. 
Table 5 Antimicrobial activity, bacterial sensitivity, intermediate sensitivity and resistance to antibiotics commonly used against Klebsiella pneumoniae and Proteus spp

\begin{tabular}{|c|c|c|c|c|c|c|c|c|c|c|}
\hline \multirow[b]{2}{*}{ Antimicrobial drug } & \multicolumn{5}{|c|}{ K pneumoniae (9 isolates) } & \multicolumn{5}{|c|}{ Proteus spp (9 isolates) } \\
\hline & $\mathrm{MIC}_{50}$ & $\mathrm{MIC}_{90}$ & $\mathbf{S} \%$ & $1 \%$ & $\mathbf{R} \%$ & $\mathrm{MIC}_{50}$ & $\mathrm{MIC}_{90}$ & $\mathbf{S} \%$ & $1 \%$ & $\mathbf{R} \%$ \\
\hline Fosfomycin tromethamine* & 1 & 32 & 100.0 & 0.0 & 0.0 & 1 & $>256$ & 55.6 & 22.2 & 22.2 \\
\hline Levofloxacin & 0.125 & $>256$ & 66.7 & 0.0 & 33.3 & 1 & 16 & 55.6 & 11.1 & 33.3 \\
\hline Moxifloxacin & 0.062 & $>256$ & - & - & - & 8 & 64 & - & - & - \\
\hline Cefdinir & 128 & 256 & 44.4 & 0.0 & 55.6 & 0.25 & 32 & 55.6 & 0.0 & 44.4 \\
\hline Cefixime & 2 & $>256$ & 44.4 & 11.2 & 44.4 & $\leq 0.016$ & 16 & 77.8 & 0.0 & 22.2 \\
\hline Cefaclor & 256 & $>256$ & 44.4 & 0.0 & 55.6 & $>256$ & $>256$ & 44.4 & 0.0 & 55.6 \\
\hline Cefprozil & $>256$ & $>256$ & 44.4 & 0.0 & 55.6 & 256 & $>256$ & 44.4 & 0.0 & 55.6 \\
\hline Cefuroxime & 64 & $>256$ & 44.4 & 0.0 & 55.6 & 128 & $>256$ & 44.4 & 0.0 & 55.6 \\
\hline Amoxicillin/clavulanic acid & 8 & 16 & 66.7 & 33.3 & 0.0 & 16 & 64 & 44.4 & 44.5 & 11.1 \\
\hline Cefotaxime & 2 & 128 & 44.4 & 11.2 & 44.4 & 0.125 & 64 & 66.7 & 0.0 & 33.3 \\
\hline Nitrofurantoin & 32 & 256 & 66.7 & 22.2 & 11.1 & 64 & 128 & 11.1 & 77.8 & 11.1 \\
\hline
\end{tabular}

${ }^{*}$ Determination of the breakpoint of fosfomycin tromethamine to Enterobacteriaceae (sensitivity rate $\leq 64 \mathrm{mg} / \mathrm{L} ;$ resistance rate $\geq 256 \mathrm{mg} / \mathrm{L}$ ).

I, intermediate; MIC, minimal inhibitory concentration; R, resistant; S, susceptible.

ESBL-negative $E$ coli isolates. We thus consider that in the treatment of complicated UTIs, antibiotics should be indicated based on susceptibility results.

Our findings showed a good antibacterial activity of fosfomycin tromethamine against both ESBL-negative and ESBL-producing $E$ coli, with bacterial sensitivity rates of $95.1 \%$ and $87 \%$ and resistance rates of $0 \%$ and $4.3 \%$, respectively. The antibacterial effect of this compound on ESBL-producing $E$ coli was slightly inferior to nitrofurantoin but superior to the other tested drugs, and consistent with previously published findings. ${ }^{24}$ In addition, a significant antibacterial activity was demonstrated against $K$ pneumoniae. However, empirical treatment with fosfomycin tromethamine and nitrofurantoin is only indicated in uncomplicated UTIs.
In our study, coagulase-negative staphylococci were the major Gram-positive pathogens identified. Initially considered as part of commensal flora and culture contaminants, since 1970 this type of staphylococci has been recognised as an aetiological agent in a variety of infections. ${ }^{25}$ The main virulence factor is mucilage polysaccharide (biofilm) production ${ }^{26}$ facilitating their adhesion to smooth surfaces (such as the uroepithelium) ${ }^{27}$ and providing resistance against antibiotics and phagocytosis. ${ }^{28}{ }^{29}$ In our study, Gram-positive bacteria were isolated more frequently in samples from cases with complicated UTIs than in samples from cases with uncomplicated UTIs. Furthermore, $S$ epidermidis was more frequently identified in women, and E faecalis was more frequently identified in men than in women. These gender differences observed in the aetiology of

Table 6 Antimicrobial activity, bacterial sensitivity, intermediate sensitivity and resistance to antibiotics commonly used against coagulase-negative Staphylococcus spp, Staphylococcus epidermidis and Enterococcus faecalis

\begin{tabular}{|c|c|c|c|c|c|c|c|c|c|c|c|c|c|c|c|}
\hline \multirow[b]{2}{*}{ Antimicrobial drug } & \multicolumn{5}{|c|}{$\begin{array}{l}\text { Coagulase-negative } \\
\text { Staphylococcus spp } \\
\text { (26 isolates) }\end{array}$} & \multicolumn{5}{|c|}{$\begin{array}{l}\text { S epidermidis } \\
\text { (16 isolates) }\end{array}$} & \multicolumn{5}{|c|}{$\begin{array}{l}\text { E faecalis } \\
\text { (15 isolates) }\end{array}$} \\
\hline & $\mathrm{MIC}_{50}$ & $\mathrm{MIC}_{90}$ & $\mathrm{~S} \%$ & $1 \%$ & $\mathbf{R} \%$ & $\mathrm{MIC}_{50}$ & $\mathrm{MIC}_{90}$ & $\mathbf{S} \%$ & $1 \%$ & $\mathbf{R} \%$ & $\mathrm{MIC}_{50}$ & $\mathrm{MIC}_{90}$ & $\mathbf{S} \%$ & $1 \%$ & $\mathbf{R} \%$ \\
\hline Fosfomycin tromethamine* & 0.25 & 32 & 96.2 & 0.0 & 3.8 & 0.25 & 0.5 & 100.0 & 0.0 & 0.0 & 32 & 32 & 100.0 & 0.0 & 0.0 \\
\hline Levofloxacin & 0.5 & 128 & 50.0 & 0.0 & 50.0 & 4 & 128 & 43.8 & 0.0 & 56.2 & 8 & 32 & 40.0 & 0.0 & 60.0 \\
\hline Moxifloxacin & 0.125 & 16 & 50.0 & 23.1 & 26.9 & 1 & 16 & 43.8 & 31.2 & 25.0 & 1 & 8 & - & - & - \\
\hline Cefdinir & 0.5 & $>256$ & 61.5 & 11.6 & 26.9 & 0.5 & $>256$ & 56.3 & 12.5 & 31.2 & 1 & $>256$ & - & - & - \\
\hline Cefixime & 16 & $>256$ & - & - & - & 16 & $>256$ & - & - & - & 8 & $>256$ & - & - & - \\
\hline Cefaclor & 4 & 64 & 69.2 & 19.3 & 11.5 & 4 & 64 & 68.8 & 18.7 & 12.5 & 64 & $>256$ & - & - & - \\
\hline Cefprozil & 1 & 32 & 88.5 & 0.0 & 11.5 & 1 & 32 & 87.5 & 0.0 & 12.5 & 8 & 64 & - & - & - \\
\hline Cefuroxime & 1 & 128 & 88.5 & 0.0 & 11.5 & 1 & 128 & 87.5 & 0.0 & 12.5 & 8 & $>256$ & - & - & - \\
\hline Amoxicillin/clavulanic acid & 0.5 & 16 & 88.5 & - & 11.5 & 0.25 & 16 & 87.5 & - & 12.5 & 0.5 & 2 & - & - & - \\
\hline Cefotaxime & 2 & 16 & 88.5 & 7.7 & 3.8 & 2 & 16 & 87.5 & 12.5 & 0.0 & 2 & $>256$ & - & - & - \\
\hline Azithromycin & 64 & $>256$ & 15.4 & 0.0 & 84.6 & $>256$ & $>256$ & 18.8 & 0.0 & 81.2 & $>256$ & $>256$ & - & - & - \\
\hline Nitrofurantoin & 16 & 32 & 100.0 & 0.0 & 0.0 & 16 & 32 & 100.0 & 0.0 & 0.0 & 32 & 32 & 100.0 & 0.0 & 0.0 \\
\hline Oxacillin & 0.5 & 128 & 38.5 & - & 61.5 & 0.5 & 128 & 37.5 & - & 62.5 & - & - & - & - & - \\
\hline
\end{tabular}


UTIs should be considered in the selection of antibiotics. In our population, susceptibility results showed that $S$ epidermidis had a high-resistance rate to levofloxacin (56.2\%), but a moderate resistance rate to second-generation and third-generation cephalosporin $(12.5 \%)$. Fosfomycin tromethamine demonstrated a larger antibacterial activity on coagulase-negative staphylococci than other tested antibiotics. For $S$ epidermidis, MIC50 and MIC90 values were 0.25 and $0.5 \mathrm{mg} / \mathrm{L}$, respectively, and sensitivity rates were up to $100 \%$. The rate of resistance of E faecalis against levofloxacin was $60 \%$, while the rate of sensitivity for fosfomycin tromethamine and nitrofurantoin was $100 \%$, with MIC values between 8 and $32 \mathrm{mg} / \mathrm{L}$. These results are consistent with those published by Perri et $a l^{30}$ who reported that 51 of the 52 Enterococcus faecium and all E faecalis isolates tested were susceptible or had intermediate susceptibility to fosfomycin.

\section{CONCLUSION}

The results of this multicenter study showed that ESBL-producing $E$ coli is the main pathogen causing symptomatic UTIs in this Chinese population. Of concern is that the resistance rate of this pathogen against commonly used antibiotics has increased. Fosfomycin tromethamine and nitrofurantoin showed a good antibacterial activity against identified pathogens, and thus can be considered for use as empirical treatment in uncomplicated UTIs. However, the mechanism on bacterial resistance is complex and diverse, and the phenomenon of multidrug-resistant bacteria has become a global burden. Susceptibility testing is a valuable tool to help in the selection of antibiotic treatment.

\section{Author affiliations}

${ }^{1}$ Department of Urology, Beijing Tongren Hospital Capital Medical University, Beijing, China

${ }^{2}$ Department of Urology, Beijing Cancer Hospital, Beijing, China

${ }^{3}$ Department of Urology, Peking University First Hospital, Beijing, China

${ }^{4}$ Institute of Clinical Pharmacology, Peking University First Hospital, Beijing, China

${ }^{5}$ Department of Urology, Peking University Shougang Hospital (Jieping Wu Urology Center), Beijing, China

${ }^{6}$ Department of Urology, Peking University People's Hospital, Beijing, China

${ }^{7}$ Department of Urology, Second Affiliated Hospital of Tianjin Medical

University, Tianjin, China

${ }^{8}$ Department of Urology, First Affiliated Hospital of China Medical University, Shenyang, China

${ }^{9}$ Department of Urology, Qilu Hospital of Shandong University, Jinan, China

${ }^{10}$ Department of Urology, Hubei Tongji Hospital, Wuhan, China

${ }^{11}$ Department of Urology, Hunan Xiangya Second Hospital, Changsha, China

${ }^{12}$ Department of Urology, Shanghai Changhai Hospital, Shanghai, China

${ }^{13}$ Department of Urology, Zhongda Hospital affiliated to Southeast University, Nanjing, China

${ }^{14}$ Department of Urology, Beijing Friendship Hospital Capital Medical University, Beijing, China

Acknowledgements The authors would like to thank all study participants and the general practitioners, study nurses and personnel who contributed to this study. They wish to acknowledge the support provided by Linyu Li and Jinghan Zhang. They also thank Juliette Gray and Adriana Rusu (XPE Pharma \& Science) for editorial support.
Contributors $S C$ and $L-D Q$ designed research and defined the research theme; L-DQ, YY, KZ, H-FG, BY, Y-JN, YW, B-KS, W-MY, X-KZ, X-FG, MC and $Y$ T performed the research. BZ carried out the laboratory experiments, $Y T$ and L-DQ analysed the data and interpreted the results. All authors participated in the writing of the article and have read and approved the manuscript.

Funding This research received no specific grant from any funding agency in the public, commercial or not-for-profit sectors.

Patient consent Obtained.

Ethics approval The Ethics Committee of Beijing Tongren Affiliated Hospital of Capital Medical University.

Provenance and peer review Not commissioned; externally peer reviewed.

Data sharing statement No additional data are available.

Open Access This is an Open Access article distributed in accordance with the Creative Commons Attribution Non Commercial (CC BY-NC 3.0) license, which permits others to distribute, remix, adapt, build upon this work noncommercially, and license their derivative works on different terms, provided the original work is properly cited and the use is non-commercial. See: http:// creativecommons.org/licenses/by-nc/3.0/

\section{REFERENCES}

1. Stamm WE, Norrby SR. Urinary tract infections: disease panorama and challenges. J Infect Dis 2001;183(Suppl 1):S1-4.

2. Foxman $\mathrm{B}$, Barlow $\mathrm{R}$, d'Arcy $\mathrm{H}$, et al. Urinary tract infection: estimated incidence and associated costs. Ann Epidemiol 2000;10:509-15.

3. Carlson KJ, Mulley AG. Management of acute dysuria: a decision analysis model of alternative strategies. Ann Intern Med 1985;102:244-9.

4. Kunin CM. Urinary tract infections in females. Clin Infect Dis 1994;18:1-10.

5. Engel JD, Schaeffer AJ. Evaluation of and antimicrobial therapy for recurrent urinary tract infections in women. Urol Clin North Am 1998;25:685-701.

6. Foxman B, Gillespie B, Koopman J, et al. Risk factors for second urinary tract infection among college women. Am J Epidemiol 2000;151:1194-205.

7. Foxman B, Frerichs RR. Epidemiology of urinary tract infection. II. Diet, clothing, and urination habits. Am J Public Health 1985;75:1314-17.

8. Foxman B. Epidemiology of urinary tract infections: incidence, morbidity and economic costs. Am J Med2002;113(Suppl 1A):5S-13S.

9. Jiangqiao W, Xueping W, Yujuan L. Nosocomial infection point-prevalence survey in three consecutive years. Chin J Infect Control 2006;5:19-34

10. Jinxian W, Xiaoling Z, Liya Y. A nosocomial infection point-prevalence survey: results and analysis. Chinese J Nosocomiol 2005;15:1119-20.

11. Talan D, Stamm W, Hooton T, et al. Comparison of ciprofloxacin (7days) and trimethoprim-sulfamethoxazole (14days) for acute uncomplicated pyelonephritis in women: a randomized trial. JAMA 2000;283:1583-90.

12. Qi HM, Lv Y, Qian X. Ministry of Health National Antimicrobial Resistant Investigation Net 2010 annual report: bacterial resistances monitor of women urine samples. Chinese $J$ Clin Pharmacol 2011;27:913-18.

13. Zheng B, Lv Y. Ministry of Health National Antimicrobial Investigation Net 2010 report: bacterial distribution and resistance in urinary tract infections in male. Chinese J Clin Pharmacol 2011;27:905-12.

14. Clinical and Laboratory Standards Institute (CLSI). Performance Standards for Antimicrobial Susceptibility Testing; Twentieth Informational Supplement. CLSI document M100-S20. Wayne: CLSI, 2011.

15. Sanders CC, Barry AL, Washington JA, et al. Detection of extended spectrum beta-lactamases producing members of family Enterobacteriaceae with the Vitek ESBL test. J Clin Microbiol 1996;34:2997-3001.

16. Lei G, Yonghong X. 2006-2007 Mohnarin report:Bacterial distribution and resistance in urinary tract infections. Chinese $J$ Antibiotics 2008;33:586-634.

17. Khan AU, Zaman MS. Multiple drug resistance pattern in Urinary Tract Infection patients in Aligarh. Biomed Res 2006;17:179-81.

18. Mishra MP, Debata NK, Padhy RN, et al. Surveillance of multidrug resistant uropathogenic bacteria in hospitalized patients in Indian. Asian Pac J Trop Biomed 2013;3:315-24. 
19. Mukherjee M, Basu S, Mukherjee SK, et al. Multidrug-resistance and extended spectrum beta-lactamase production in uropathogenic E. Coli which were isolated from hospitalized patients in Kolkata, India. J Clin Diagn Res 2013;7:449-53.

20. Odongo CO, Anywar DA, Luryamamoi $\mathrm{K}$, et al. Antibiograms from community-acquired uropathogens in Gulu, northern Uganda-a cross-sectional study. BMC Infect Dis 2013;13:193.

21. Guangying $Y$, Xueyin $Z$, Anrong $L$. Development of a novel test identifying extended-spectrum beta lactamase producing strains relative to resistant plasmids. Chin J Microecol 2002; 14:89-90.

22. Hua Y, Hua L, Yingjun Y. Distribution of pathogens and resistance detection in urinary infection. Chinese J Nosocomiol 2003;13:982-4.

23. Haishen K, Baoguan W, Yi G. Drug resistance of Pneumonia klebsiella and $E$. coli to ultra wide spectrum Beta-lactam enzyme. Chinese J LabMed 2000;1:23-5.
24. Maraki S, Samonis G, Rafailidis PI, et al. Susceptibility of urinary tract bacteria to fosfomycin. Antimicrob Agents Chemother 2009;53:4508-10

25. Piette A, Verschraegen G. Role of coagulase-negative staphylococci in human disease. Vet Microbiol 2009;134:45-54.

26. Huebner J, Goldmann DA. Coagulase-negative staphylococci: role as pathogens. Annu Rev Med 1999;50:223-36.

27. O'Gara JP, Humphreys H. Staphylococcus epidermidis biofilms: importance and implications. J Med Microbiol 2001;50:582-7.

28. Costerton JW, Stewart PS, Greenberg EP. Bacterial biofilms: a common cause of persistent infections. Science 1999;284:1318-22.

29. Mack D, Davies AP, Harris LG, et al. Microbial interactions in Staphylococcus epidermidis biofilms. Anal Bioanal Chem 2001;387:399-408.

30. Perri $\mathrm{MB}$, Hershberger $\mathrm{E}$, lonescu $\mathrm{M}$, et al. In vitro susceptibility of vancomycin-resistant Enterococci (VRE) to fosfomycin. Diagn Microbiol Infect Dis 2002;42:269-71a. 